\title{
La Comisión Nacional de Energía Atómica y la consolidación del complejo empresarial en torno a la actividad nucleoeléctrica (1976-1994)
}

\author{
The National Atomic Energy Commission \\ and the consolidation of the business complex \\ around nuclear activity (1976-1994)
}

\author{
Milagros Rodríguez \\ Universidad de Buenos Aires \\ (Argentina) \\ mirodriguez@filo.uba.ar
}

\begin{abstract}
Resumen
El objetivo de este trabajo consiste en analizar las características que adquirió la consolidación de empresas mixtas en torno a la generación nucleoeléctrica por iniciativa de la Comisión Nacional de Energía Atómica (CNEA). Dicha política se hallaba en estrecha relación con la noción de desarrollo científico tecnológico autónomo y la necesidad de promover la participación de proveedores locales en el sector nucleoeléctrico a fin de fortalecer la industria nacional. Por este motivo, desde 1976 la institución venía llevando a cabo una política de entrega al sector privado y provincial de ciertas actividades ligadas a la producción de insumos para la industria nuclear. La conformación de empresas se realizó, en cada caso, bajo diversas formas jurídicas y distintos grados de participación para optimizar su gestión.
\end{abstract}

Palabras Clave: Estado empresario - Comisión Nacional de Energía Atómica - Industria Nuclear 


\begin{abstract}
The aim of this paper is to analyze the characteristics of consolidation of joint ventures around nuclear power generation, as an initiative of the Comisión Nacional de Energía Atómica (CNEA). This policy was closely related to the notion of autonomous scientific and technological development and the need to promote the participation of local suppliers in the nuclear power sector in order to strengthen the domestic industry. For this reason, since 1976 the institution had been carrying out a policy of delivery to the private and provincial sector certain activities linked to the production of materials for the nuclear industry. The formation of companies was performed in each case, under different legal forms and varying degrees of participation to optimize management.
\end{abstract}

Key Words: State Employer - Comisión Nacional de Energía Atómica - Nuclear industry

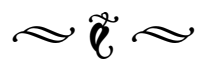

\section{Introducción}

En la actualidad, los estudios relacionados con el sector nuclear en nuestro país, constituyen un corpus bibliográfico incompleto $y$ fragmentario. Gran parte del mismo se compone de trabajos, testimonios y relatos realizados por científicos -provenientes del ámbito de la física y la química- que participaron activamente de las actividades de la Comisión. En tanto, la producción académica desde la disciplina histórica se encuentra aún en sus primeras fases, siendo la etapa que va desde la creación de la Comisión hasta la construcción del reactor RA-1 la más estudiada. ${ }^{1}$

Por ende, a más de 60 años de su existencia, resulta llamativo el descuido en torno a las múltiples aristas de la cuestión por parte de la comunidad académica. Dicha característica se

1. Para un completo estado de la cuestión véase RODRÍGUEZ, Milagros, "Cuentas Pendientes. La producción historiográfica argentina en torno a la generación nucleoeléctrica", ponencia presentada en $X L I$ Reunión Anual de la AATN, Ministerio de Relaciones Exteriores y Culto, Buenos Aires, 2014. inscribe en un panorama latinoamericano más amplio, signado por la ausencia de una correcta articulación en los sistemas nacionales de Ciencia y Tecnología (a partir de ahora, CyT). De esta forma, la tarea urgente de reconstruir esa trayectoria a fin de diseñar políticas públicas de mayor alcance y eficiencia, ha sido destacada por algunos autores especializados en la materia. ${ }^{2}$

Por otra parte, desde la década de los '90, la difusión de trabajos provenientes del "think thank" neoliberal instauraron en el sentido común la idea de que las empresas públicas argentinas resultan incapaces de incorporar desarrollos tecnológicos para brindar bienes y servicios de alto valor agregado. ${ }^{3}$ En líneas

2. HURTADO DE MENDOZA, Diego, La ciencia argentina. Un proyecto inconcluso: 1930-2000, Edhasa, Buenos Aires, 2010, pp. 30 y OTEIZA, Enrique "Introducción" en OTEIZA, Enrique; (et al.) La politica de investigación científica y tecnológica argentina. Historia y perspectivas, CEAL, Buenos Aires, 1992, pp. 11.

3.Al respecto véase: RAMÍREZ, Hernán, Corporaciones en el poder. Institutos económicos y acción politica en Brasil y Argentina: IPESS, FIEL y Fundación Mediterránea, Lenguaje Claro, Buenos Aires, 2007 y SARALE, 
generales, aquel discurso propone que la creación de industrias "artificiales" beneficiadas por las políticas de industrialización sustitutiva no han sabido desarrollar la capacidad de diversificar su producción e integrarse en el mercado externo. Cabe destacar que estas ideas fueron difundidas por un grupo de economistas liberales educados en la Universidad de Chicago bajo la dirección de figuras como Milton Friedman y Arnold Harberger. Apoyados por poderosos organismos internacionales $y$ empresas multinacionales que hacían suyos los postulados del consenso de Washington, dejaron sentir su influencia a través de institutos como la Fundación Mediterránea, el Centro de Estudios Macroeconómicos Argentino (CEMA) y la Fundación de Investigaciones Económicas Latinoamericanas (FIEL). ${ }^{4}$

Sin embargo, hacia mediados de la década de los '90, algunos estudios han revelado que aquel contexto de liberalización y apertura, permitió la configuración de ámbitos de negocios que dieron lugar en nuestro país una nueva generación de empresas. Dentro de sectores económicos específicos, estos trabajos observan trayectorias que describen una tendencia creciente a la innovación y la internacionalización. ${ }^{5}$ En esta línea destaca

Gustavo, Poder económico y economistas: El papel de la Fundación Mediterránea, la FIEL y el CEMA en la construcción de la hegemonía neoliberal en Argentina, 2006. Disponible en: <http://www.fcp.uncu.edu.ar/upload/ PODERECONOMICOYECONOMISTAS.PDF> [Consulta: 15 de Mayo 2014].

\section{FIEL, El fracaso del estatismo. Una propuesta para} la reforma del sector público argentino, Sudamericana/ Planeta, Buenos Aires, 1987, p.12-13; PIÑEIRO, José, y ZOTHNER, Hugo, "Análisis del Proceso Privatizador Argentino. Sector Empresas Públicas no Financieras”, en Revista ASAP, Buenos Aires, 1994, No 24, p. 57.

5. KOSACOFF, Bernardo (editor) Estrategias empresariales en tiempos de cambio. El desempeño industrial la compilación realizada por Hernán Thomas, Guillermo Santos y Mariano Fressolli, titulada "Innovar en Argentina". La misma aporta una serie de trabajos que desmienten la ausencia de innovaciones empresariales a través del análisis de seis trayectorias exitosas basadas en la incorporación intensiva de tecnologías. ${ }^{6}$ Lo novedoso de la selección es que abarca empresas de distinto tipo -privado, estatal y mixto- cuya trayectoria puede rastrearse en muchos casos desde las décadas de los ' $60 \mathrm{y}$ $\operatorname{los}{ }^{\prime} 70$.

En el marco de aquellas investigaciones, el objetivo de este trabajo consiste en analizar las características que adquirió la consolidación de empresas mixtas en torno a la generación nucleoeléctrica por iniciativa de la Comisión Nacional de Energía Atómica (a partir de ahora, CNEA). Dicha política se hallaba en estrecha relación con la noción de desarrollo científico tecnológico autónomo y la necesidad de promover la participación de proveedores locales en el sector nucleoeléctrico a fin de fortalecer la industria nacional. Es así que desde la creación de INVAP en 1976 hasta la reforma administrativa de todo el sector en 1994, la institución desarrolló una activa política de entrega al sector privado y provincial de actividades ligadas a la producción de insumos para la industria nuclear.

A través del análisis de fuentes diversas se procederá a responder los siguientes interrogantes ¿Qué motivos impulsaron a la CNEA a delegar ciertas actividades

frente a nuevas incertidumbres, Universidad Nacional de Quilmes/CEPAL, Bernal, 1998.

6. THOMAS, Hernán, SANTOS, Guillermo y FRESSOLI, Mariano, Innovar en Argentina. Seis trayectorias empresariales basadas en estartegias intensivas en conocimiento, Lenguaje Claro, Carapachay, 2013. 
productivas? ¿Bajo qué condiciones? ¿Qué papel desempeñaron las nuevas entidades en torno al Plan Nuclear de 1979? ¿De qué forma se vieron afectadas por la paralización del mismo durante la etapa neoliberal? ¿Qué estrategias implementaron? ¿Lograron diversificarse para brindar bienes y servicios hacia otras actividades productivas del país?

\section{CNEA: vinculación entre empresas y desarrollos científicos}

A diferencia de otras instituciones de $\mathrm{CyT}$ en nuestro país, desde mediados de los años ' 70 , la CNEA comenzó a concretar una política de entrega de áreas productivas al sector privado o provincial. La institución, creada por el gobierno peronista en 1950 con el objetivo de coordinar, estimular y controlar todas las investigaciones en el campo nuclear, se hallaba por entonces avocada a la construcción de las primeras Centrales Nucleares de potencia en el país. ${ }^{7}$

Para explicar las motivaciones que guiaban dicha política, cabe reseñar la cuestión de la temprana vinculación entre el mundo empresarial y el ámbito científico y tecnológico de la CNEA. Desde fines de la década del '50, Jorge Alberto Sabato había destacado la importancia de la tecnología como herramienta para el desarrollo, motivo por el cuál consideraba sumamente necesario que los avances científicos se transfieran hacia la industria nacional. Dicho esquema alcanzaría luego su forma más acabada en la formulación del "Triángulo de Sabato"; en cada uno de los vértices de la figura, el autor ubicaba al gobierno,

7. DECRETO LEY 10936/50, Boletín Oficial, 30 de Mayo de 1950.

72 la estructura productiva y la infraestructura científica. La finalidad era ilustrar la correcta integración que debía lograrse entre las tres partes. Asimismo, señalaba que en nuestro país, la constitución de un complejo de CyT maduro requería de la solución a dos cuestiones. Por un lado, la mejora de la infraestructura y por el otro, la incorporación del conocimiento a la producción para saber cómo y dónde innovar. ${ }^{8}$

Siguiendo estas ideas, Sabato creó en 1955 el Departamento de Metalurgia dentro de la CNEA, con la finalidad de aprovisionar al sector pero también de comenzar a instalar en el país capacidades que pudieran ser aprovechadas por otras industrias. ${ }^{9} \mathrm{~A}$ su vez, el Instituto era dotado de cierta autonomía en función de posibilitar el desarrollo de la metalurgia fuera del ámbito nuclear. ${ }^{10}$

Siguiendo la misma línea de razonamiento, en 1962, Sabato creó el Servicio de Asistencia Técnica a la Industria (SATI) para activar la participación industrial en el desarrollo científico. Su estructura era concebida como una entidad sin fines de lucro, dado que el objetivo principal de la institución apuntaba a derramar el conocimiento derivado del

8. BOTANA, Natalio, SABATO, Jorge, "La ciencia y la tecnología en el desarrollo futuro de América Latina", en SABATO, Jorge (compilador) El Pensamiento Latinoamericano el la problemática Ciencia, Tecnologia, Desarrollo, Dependencia, Paidós, Buenos Aires, 1975, pp. 143-154.

9. Véase SÁBATO, Jorge, "Quince años de metalúrgia en la Comisión Nacional de Energía Atómica [Entrevista]", en Ciencia Nueva, 1973, N 15, pp. 1-19.

10. ENRIQUEZ, Santiago, "A 50 años del Servicio de Asistencia Técnica a la Industria (SATI): Apuntes de una heterodoxia.", en Revista de la CNEA,Buenos Aires, 2011, V.XI, N 41-42, p. 16. Disponible en:<http:// www.iaea.org/inis/collection/NCLCollectionStore/_ Public/46/135/46135001.pdf> 
desarrollo nuclear en las empresas nacionales. Si bien la estrategia se restringía a una rama particular, el éxito de la misma se manifestó en la creciente participación de la industria nacional en la construcción de Atucha I y Embalse. De esta forma, el circuito se retroalimentaba; los conocimientos científicos aportados a la industria por la CNEA eran "retribuidos" a través de la oferta de productos y servicios sumamente valiosos para el Plan Nuclear y las Centrales. ${ }^{11}$

En este contexto se inscribe la concreción de emprendimientos mixtos de la CNEA con capitales privados y provinciales. Entre 1976 -año de la creación de la primera empresay 1994 -fecha de la reforma administrativa de la institución-, podemos diferenciar dos etapas en las cuales el contexto económico jugó un rol fundamental a la hora de explicar las motivaciones que impulsaban la política de creación de emprendimientos mixtos practicada por la CNEA. Mientras que durante los años del último gobierno de facto la institución gozó de un lugar preponderante en el panorama institucional argentino, la cuestión revistió un carácter estratégico asociado a los lineamientos del Plan Nuclear de 1979. En cambio, las restricciones financieras acaecidas a partir del retorno de la democracia replantearon seriamente los objetivos de dicha política, ahora en pos de escapar del estrangulamiento presupuestario.

\section{La creación de empresas durante la última dictadura militar (1976-1984)}

Para 1976 el modelo de Industrialización por Sustitución de Importaciones evidenciaba su 11. Ídem, pp. 17-18. agotamiento en una crisis profunda. Como respuesta, la postura oficial del gobierno militar elaboró un diagnóstico sumamente reactivo hacia los pilares que sustentaban el modelo sustitutivo. Según el Ministro de Economía, Martínez de Hoz, el déficit presupuestario y la inflación eran distorsiones producidas por la intervención estatal. Las mismas debían ser eliminadas mediante la "eficiencia" en la asignación de los recursos y el establecimiento de los precios mediante la libre competencia y completa certidumbre. ${ }^{12}$ En consonancia con ello, el "Estado Empresario" debía transformarse en un "Estado Subsidiario": el poder público solo debía mantener bajo su órbita áreas cuya privatización resultara inconveniente oimposible.Comoconsecuencia, gran cantidad de empresas estatales fueron sometidas a un proceso de "privatización periférica”, y delegadas contractualmente a poderosos grupos económicos privados. ${ }^{13}$ En cuanto a la industria, se objetó definitivamente su capacidad para motorizar el desarrollo económico. Si bien la política privatizadora no tuvo un efecto homogéneo, sino que afectó fundamentalmente a pequeñas y medianas empresas, consolidó el poder de poderosos grupos económicos locales y extranjeros. ${ }^{14}$

12. CANITROT, Adolfo, "La disciplina como objetivo de la política económica. Un ensayo sobre el programa económico del gobierno argentino desde 1976", en Desarrollo Económico, Buenos Aires, 1980, V.XIX, № 76, p. 470.

13. BELINI, Claudio y ROUGIER, Marcelo, El Estado Empresario en la Industria Argentina. Conformación y crisis, Manantial, Buenos Aires, 2008, p. 265.

14. SCHORR, Martin, Industria y Nación.

Poder económico, neoliberalismo y alternativas de reindustrialización en la Argentina contemporánea, Edhasa, Buenos Aires, 2004, pp. 65-66. 
En abierta contradicción con la implementación de un nuevo modelo de acumulación basado en la valorización financiera de la economía, la CNEA empezaba a constituirse como un espacio privilegiado al amparo de la cual se realizaban importantes avances en materia de incorporación de innovaciones y desarrollos autónomos.

A partir de mediados de 1980, se pusieron en marcha los grandes proyectos delineados por el Plan Nuclear, la prospección y explotación de yacimientos de uranio, la construcción de Atucha II -la primera de las cuatro centrales programadas-, la construcción de una planta piloto de enriquecimiento de uranio y la construcción de una planta industrial de agua pesada. El cronograma preveía el autofinanciamiento del Plan a través de la fijación de tarifas eléctricas para la venta de energía. En el caso de la Central Atucha II, la mitad de la inversión provino de préstamos acordados contractualmente con Siemens, mientras que el $50 \%$ restante era financiado por el Estado Argentino. Hacia 1982, la CNEA alcanzó los niveles presupuestarios más altos de su historia, llegando a representar casi el $2 \%$ del PBI. ${ }^{15}$

En continuidad con las ideas de Sabato, la CNEA comenzaba a encarar por esos años una ambiciosa política de conformación de empresas mixtas con distintas entidades. Se planteaba entonces que los avances científicos y tecnológicos realizados por la Comisión en cada una de las actividades productivas del ciclo de combustible serían transferidos a capitales de origen privado y provincial. Cabe destacar que este modelo configuraba una

15. CNEA, "La política nuclear Argentina. Evaluación y propuestas de la Comisión Nacional de Energía Atómica”, en Revista CNEA, Buenos Aires, 2001, pp. 6-9. alternativa al proceso de privatización que finalmente predominó en los años ‘ 90 y que no fue tenida en cuenta por el gobierno de turno a pesar de que había demostrado ser exitosa.

A lo largo de su historia, la CNEA participó en la fundación de diferentes tipos de organizaciones y empresas con funciones específicas en el ciclo de producción energía nuclear. De esta forma, se fue consolidando con el correr de los años un polo empresarial en torno a la institución, mediante la asociación de capitales públicos y privados y con distintos grados de participación accionaria.

Según las memorias emitidas por la institución, la finalidad última de dicha política consistía en:

(1) Promover la regionalización de la actividad nuclear y crear fuentes de trabajo genuinas,

(2) Asignar al capital privado un rol preponderante en algunas áreas de la producción para agilizar el manejo y la favorecer la gestión empresarial y;

(3) Liberarse de normas administrativas estatales que imponían algunas limitaciones a la flexibilidad de la gestión, como la fiscalización del Tribunal de Cuentas de la Nación.

El primer objetivo se concretó a través del convenio entre la Comisión y distintos Estados provinciales de la República Argentina, sobre todo de la región de Cuyo. En cuanto al segundo y tercer objetivo, los miembros de la institución reconocían explícitamente que existían ciertas limitaciones en la capacidad operativa de la gestión pública para este tipo de actividades. El ex director de la CNEA, Vicealmirante Carlos 
Castro Madero, comentaba que "El sector privado debe poder acceder a los segmentos productivos de la actividad nuclear y contribuir a su financiamiento... El objetivo de la CNEA debe ser reducir al máximo el costo de generación de kwh nuclear, para mejorar el nivel de vida general y reducir el costo de los productos manufacturados, necesidad más acuciante cuando se piensa en exportar" ${ }^{16}$

Por otra parte, el dominio de todas las etapas del ciclo de combustible aseguraría una importante cuota de autonomía en la toma de decisiones respecto de los insumos utilizados para abastecer a las centrales nucleares. ${ }^{17}$ Este punto resultaba sumamente relevante si tenemos en cuenta que por esos años las presiones ejercidas desde el Club de Londres por desarmar el Plan Nuclear Argentino habían alcanzado niveles alarmantes. ${ }^{18}$ Siguiendo este

16. CASTRO MADERO, Carlos y TAKACS, Esteban, Politica nuclear argentina i avance o retroceso?, Instituto de publicaciones navales, Buenos Aires, 1991, p.25.

17. HURTADO DE MENDOZA, Diego, El sueño de la Argentina Atómica. Política, tecnología nuclear y desarrollo nacional (1945-2006), Edhasa, Buenos Aires, 2014, p. 108.

18. Organizado como un pool nuclear informal, el "Club de Londres" había nacido bajo la iniciativa estadounidense en 1974 con el objetivo explícito de restringir la venta de tecnología en esa materia a fin de evitar el surgimiento de otros competidores. El Tratado de No Proliferación Nuclear, abierto a la firma en 1968 y rechazado sucesivamente por los gobiernos argentinos hasta 1995, se transformó en la punta de lanza de una puja por la defensa de la autonomía nacional. En efecto, el tratado aspiraba a limitar la cantidad de países en posesión de armas nucleares a los ya existentes (Estados Unidos, Francia, Gran Bretaña, Rusia y China). A este fin, ciertas cláusulas del mismo resultaban discriminatorias y frenaban el desarrollo pacífico de la tecnología en el resto de los países. Durante la última dictadura militar, la Argentina había reafirmado enérgicamente su negativa a firmar el tratado, pese a las presiones ejercidas por los Estados Unidos. razonamiento teórico, a lo largo de su historia la CNEA conformó dos tipos de empresas. En primer lugar, empresas de producción. Se trataba de empresas asociadas al capital privado que tenían asignada la elaboración de un producto necesario para el ciclo de combustible. Ejemplos de este tipo son: Fabricación de Aleaciones Especiales (FAE S.A.) y Combustibles Nucleares Argentinos (CONUAR S.A.). En segundo lugar, empresas de tecnología o de servicios tecnológicos, las cuales se dotaron de gran versatilidad para responder rápidamente a circunstancias $\mathrm{y}$ necesidades diversas. Entre ellas podemos nombrar: Investigaciones Aplicadas (INVAP S.A.) o Alta Tecnología (ALTEC S.E.).

De esta forma, la institución delegaba áreas específicas del circuito productivo con un porcentaje significativo de participación que aseguraba cierta coherencia en la toma de decisiones de todo el sector en conjunto y, por otra parte, garantizaba que su operación se realizara de forma segura. Una de las ventajas de este modelo, radicaba en que no se producirían desbalances entre una institución signada por la actividad científica y las empresas libradas a la lógica de mercado. Sin embargo, no en todos los casos la institución participaba mediante la retención de un porcentaje del capital accionario. En varias empresas, fundamentalmente aquellas constituidas junto con organismos provinciales, la CNEA solo se reservaba la prerrogativa de designar a los miembros del Directorio. De esta forma, tanto sea a través de la participación accionaria o no, la CNEA podía coordinar la producción de todas las esferas, así como también gestionar ágilmente recursos humanos y tecnologías. 


\section{La creación de empresas luego del retorno de la democracia (1984-1994)}

Años más tarde, el gobierno de Alfonsín asumía la gestión luego de uno de los períodos más oscuros que el país había sufrido hasta entonces en materia de derechos humanos y gestión financiera. El triste desenlace en Malvinas y la cuestión de los desaparecidos, obligaba al gobierno a concentrar todas sus energías en dos tareas fundamentales, la neutralización política de las Fuerzas Armadas para asegurar la democracia y el juicio a los culpables del terrorismo de Estado. Por otra parte, la situación económica del país que el radicalismo heredaba, se encontraba sumamente deteriorada por la política monetarista aplicada en los últimos años. El crecimiento desmedido de la deuda pública y la inflación, condujeron al país a una grave crisis, política y económica, que redujo sustancialmente la capacidad operativa del gobierno.

Lejos de revertir el modelo de acumulación basado en la financiarización de la economía, el retorno a la democracia a partir de 1983 significó la agudización del proceso. El Estado se transformó en el principal agente de reasignación de los excedentes hacia los grupos económicos más concentrados. ${ }^{19}$

En este contexto, las instituciones argentinas ligadas al sector de CyT fueron intervenidas con el fin de normalizar y democratizar sus estructuras burocráticas. Así, la desmilitarización de algunos ámbitos tradicionalmente ligados a las Fuerzas Armadas resultó prioritaria. Por otra parte,

19. Ídem, p. 69. las aéreas de atención prioritaria fueron redefinidas, poniendo el foco en la electrónica, la biotecnología y la medicina en detrimento de aquellas que tradicionalmente habían gozado mayor preponderancia, como la energía nuclear y la industria espacial. ${ }^{20}$

Como consecuencia de las circunstancias antes reseñadas, la CNEA se vio atravesada por tres nuevas tendencias que determinarían el curso de aquella década: el éxodo científico, la desmilitarización de la institución, y el recorte presupuestario que obligaba a redimensionar el Plan Nuclear heredado de la dictadura.

En cuanto a la creciente injerencia de las Fuerzas Armadas en las instituciones burocráticas del Estado, perpetrada a través de las dictaduras que se sucedieron desde la década de 1930, la gestión de Alfonsín debía encarar un programa que asegurara la subordinación definitiva a las autoridades constitucionales. Luego del terrorismo de Estado, la creciente hostilidad de la sociedad civil hacia los militares de todas las armas debía ser encauzada para normalizar el funcionamiento de la institución. El gobierno radical inició la reestructuración de las FFAA, con la finalidad de reinscribirlas en el Estado, recuperando los valores de la democracia y el profesionalismo. ${ }^{21}$ Sibien uno de los principales mecanismos fue la Ley de Defensa Nacional de 1988, también se procedió a desvincular determinadas instituciones del tradicional

20. ALBORNOZ, Mario y GORDON, Ariel, "La política de ciencia y tecnología en Argentina desde la recuperación de la democracia (1983-2009)", en ALBORNOZ, Mario y JESÚS Sebastián (editores) Trayectorias de las políticas cientificas y universitarias de Argentina y España, CSIC, Madrid, 2011, p. 12.

21. FREDERIC, Sabina, Las trampas del pasado: las Fuerzas Armadas y su integración al Estado democrático en Argentina, Fondo de Cultura Económica, Buenos Aires, 2013, pp. 53-55. 
dominio militar. En tanto la Comisión constituía un caso emblemático de gestión por parte de la Marina desde su nacimiento, el Dr. Alfonsín rompió con la tradición y designó, por primera vez, a un civil para el Directorio. Se trataba de Alberto Constantini, ingeniero civil recibido de la Universidad de La Plata, que había ocupado varios cargos públicos en el Ministerio de Obras Públicas y Servicios. A partir de aquel entonces, ningún director de la institución volvería a provenir del ámbito militar.

En segundo lugar, las restricciones financieras -en las que sin ninguna duda las presiones internacionales jugaron un papel determinantejaquearon al gobierno democrático desde el comienzo. Dicha situación hacía necesario aplicar un "reordenamiento" o "adaptación" del Plan Nuclear heredado a la nueva realidad argentina. Para 1985, era claro que los planes proyectados en 1979 se alejaban cada vez más del horizonte de lo posible. En consecuencia, el presupuesto de la CNEA fue reducido a su tercera parte, generando la progresiva paralización de las obras en curso y la contracción de la actividad en todo el sector. ${ }^{22}$

Por último, la reducción presupuestaria aceleraría el éxodo científico dentro de la institución. La pérdida de recursos humanos capacitados constituía una característica endémica del complejo de CyT argentino desde, por lo menos, 1966. La desinversión sufrida por el sector, y su contraparte en la reducción de los sueldos, sumada a la política represiva aplicada por los diversos gobiernos militares que se sucedieron desde aquella fecha, contribuyeron a profundizar el fenómeno

22. HURTADO DE MENDOZA, Diego, El sueño de la Argentina Atómica..., Op. Cit., p. 267. del "Brain Drain". ${ }^{23}$ Con el retorno de la democracia, el elenco profesional de la CNEA se redujo y experimentó un significativo envejecimiento.

Dadas las circunstancias, comenzó a plantearse en la institución la necesidad de delegar otras etapas del ciclo de combustible que habían alcanzado un grado de desarrollo tecnológico considerable, como la producción del concentrado de uranio o la gestión de residuos de alta actividad. Lo mismo debía ocurrir con las actividades de investigación y desarrollo, y los servicios técnicos y no técnicos. En definitiva, se planteaba una división del trabajo en nuevas empresas a partir de todas las tareas desempeñadas por la CNEA.

Para que la nueva estructura propuesta funcionara, la Comisión delineaba pautas específicas que reglamentaran la interacción entre todas las empresas del conglomerado: el respeto de la autoría intelectual de cada contraparte, la regulación del intercambio de recursos humanos, la coordinación entre empresas para impedir la duplicación de estructuras y asegurar el suministro de cada una de ellas, la elaboración de planes de trabajo globales que maximicen el desempeño del complejo, la fijación de pautas de comportamiento empresario y la obligatoriedad de subcontratar empresas del sistema por sobre otros oferentes.

Con respecto a las Centrales Nucleares, desde fines de la década de 1980 podemos comprobar

23. Véase OTEIZA, Enrique, "Drenaje de Cerebros. Marco histórico y conceptual", en REDES, Bernal,1996, V. III, $\mathrm{N}^{\circ} 7$, pp. 101-120. Disponible en: <http://iec.unq. edu.ar/images/redes/RedesN07/Artculos/Perspectivas/ Drenaje\%20de\%20cerebro\%20Marco\%20histrico\%20 y\%20conceptual\%20Enrique\%20O.pdf>. 
la aparición de una discusión dentro de la institución acerca dela conveniencia de trasladar su operación y gestión a manos privadas. Desde el punto de vista lógico, representaba una continuación con la política seguida para todo el ciclo de combustible, en el cual las Centrales constituían el eslabón fundamental. Sin embargo, existían limitaciones de índole económica que obstaculizaban aquel proyecto. $\mathrm{El}$ problema central radicaba en que una parte sustancial de los ingresos de la Comisión provenía de la venta de la energía producida por las Centrales Nucleares. Entre 1986 y 1988, la facturación correspondiente a Atucha I representaba en promedio alrededor de 25 millones de dólares anuales, mientras que la Central Embalse ascendía a más de $65 .{ }^{24}$ Esos ingresos representaban entre un 15\% y un $20 \%$ del presupuesto total de la institución y se destinaban a financiar investigaciones. Dadas las circunstancias de ajuste iniciadas a mediados de los ' 80 y la ausencia de un mecanismo de asignación presupuestaria alternativo que asegurara el sostenimiento de las actividades usuales de la Institución, el pasaje de la operación de las Centrales a manos privadas continuaría siendo un mero ejercicio teórico.

La llegada del neoliberalismo al poder en 1989 significó la profundización de la marginalización del desarrollo del sector manufacturero nacional iniciado en 1976. La política agresiva de privatizaciones sumada al ingreso abrupto de capitales y mercancías del exterior destruyó a la industria local cimentada en el período previo. Según Martín Schorr, este proceso de "desindustrialización" se

24. Promedio calculado en base a: CNEA, Memoria Anual 1986 y 1987, CNEA, Buenos Aires, 1988, p. 14; CNEA. Memoria Anual 1988, CNEA, Buenos Aires, 1989, p. 10. caracterizó por la reducción de la cantidad y el tamaño de las unidades productivas -con la consecuente expulsión de mano de obra y la ruptura de los encadenamientos productivosel surgimiento de un sector industrial más desarticulado y la reversión de los procesos de integración sectorial, el incremento del déficit comercial sectorial la desaparición de ramas industrias complejas, el deterioro de los precios industriales frente a los servicios $-\mathrm{y}$, por ende, la caída del peso de las manufacturas en el Producto Bruto Nacional-, el déficit comercial y la valorización financiera, que se tradujo en la transferencia de capitales al exterior. Aunque el nuevo modelo no poseía un sesgo anti-industrialista explícito, el crecimiento de los oligopolios poco dinámicos asociados a ventajas comparativas o rentas de cuasi privilegio significó la destrucción de la pequeña y mediana empresa. ${ }^{25}$

Para 1994, la Comisión había logrado conformar cinco empresas mediante acuerdos provinciales y tres de capital mixto en consorcio con la gestión privada (Cuadro 1).

A partir de mediados de la década de 1990, la paralización del Plan Nuclear y la reorganización administrativa de la CNEA, puso en jaque la continuidad misma del complejo empresarial creado por la institución desde 1976. En este sentido, el decreto 1540/94 promulgado en 1994 constituyó un hito en la concepción del rol que la CNEA y las empresas asociadas debían ocupar en el modelo de país.

E1 documento legal, elaborado por el ministro de Economía Domingo Cavallo y la Fundación Mediterránea, presentaba un plan

25. Ídem, p. 80. 


\section{Cuadro 1: Empresas creadas por la CNEA (1976-1994)}

\begin{tabular}{|c|c|c|c|c|c|c|}
\hline $\begin{array}{l}\text { Fecha de } \\
\text { Creación }\end{array}$ & Empresa & $\begin{array}{l}\text { Tipo } \\
\text { Capital }\end{array}$ & $\begin{array}{l}\text { Participación } \\
\text { CNEA }\end{array}$ & $\begin{array}{c}\text { Participación } \\
\text { Privada/ } \\
\text { Provincial }\end{array}$ & & Actividad \\
\hline 08/09/1976 & $\begin{array}{c}\text { Investigaciones Aplicadas S.A. } \\
\text { (INVAP) }\end{array}$ & Estatal & (1) (2) & $100 \%$ & $\begin{array}{c}\text { Provincia de Río } \\
\text { Negro }\end{array}$ & $\begin{array}{l}\text { Investigación y } \\
\text { desarrollo }\end{array}$ \\
\hline 07/09/1977 & Nuclear Mendoza S.E. & Estatal & (1) & $100 \%$ & $\begin{array}{l}\text { Provincia de } \\
\text { Mendoza }\end{array}$ & $\begin{array}{l}\text { Prospección y } \\
\text { Explotación de } \\
\text { minerales }\end{array}$ \\
\hline 08/07/1980 & $\begin{array}{c}\text { Empresa Nuclear Argentina } \\
\text { de Centrales Eléctricas S.A. } \\
\text { (ENACE) }\end{array}$ & Mixta & $75 \%$ & $25 \%$ & Siemens KWU & $\begin{array}{l}\text { Ingeniería de } \\
\text { centrales nucleares }\end{array}$ \\
\hline $26 / 10 / 1981$ & $\begin{array}{c}\text { Combustibles Nucleares } \\
\text { Argentinos S.A. (CONUAR } \\
\text { S.A.) }\end{array}$ & Mixta & $33 \%$ & $67 \%$ & $\begin{array}{l}\text { Grupo Pérez } \\
\text { Companc }\end{array}$ & Pastillas de uranio \\
\hline 05/02/1985 & Alta Tecnología S.E. (ALTEC) & Estatal & (1) & $100 \%$ & $\begin{array}{c}\text { Provincia de Río } \\
\text { Negro }\end{array}$ & $\begin{array}{l}\text { Informática / } \\
\text { microcomputadoras }\end{array}$ \\
\hline $30 / 04 / 1986$ & $\begin{array}{l}\text { Fabricación de Aleaciones } \\
\text { Especiales S.A. (FAE S.A.) }\end{array}$ & Mixta & $32 \%$ & $68 \%$ & $\begin{array}{c}\text { Combustibles } \\
\text { Nucleares } \\
\text { Argentinos S.A. }\end{array}$ & $\begin{array}{l}\text { Productos de } \\
\text { zircaloy }\end{array}$ \\
\hline $30 / 10 / 1986$ & $\begin{array}{c}\text { Córdoba Alta Tecnología S.E. } \\
\text { (CORATEC) }\end{array}$ & Estatal & (1) & $100 \%$ & $\begin{array}{l}\text { Provincia de } \\
\text { Córdoba }\end{array}$ & $\begin{array}{l}\text { Radioisótopos y } \\
\text { radiaciones }\end{array}$ \\
\hline $21 / 12 / 1989$ & $\begin{array}{c}\text { Empresa Neuquina de Servicios } \\
\text { de Ingeniería S.E. (ENSI) }\end{array}$ & Estatal & $49 \%$ & $51 \%$ & $\begin{array}{l}\text { Provincia de } \\
\text { Neuquén }\end{array}$ & Agua pesada \\
\hline 12/11/1996(3) & DIOXITEK S.A. & Estatal & $99 \%$ & $1 \%$ & $\begin{array}{l}\text { Provincia de } \\
\text { Mendoza }\end{array}$ & Dióxido de Uranio \\
\hline
\end{tabular}

(1) A pesar de no poseer capital accionario, CNEA posee participación en el Directorio.

(2) En el caso de INVAP, la participación de CNEA en el Directorio es mayoritaria y posee la prerrogativa de designar al Presidente y Vicepresidente de la Institución.

(3) La planta Industrial fue instalada en 1982 y transferida a Dioxitek en 1997

Fuente: elaboración propia en base a CNEA. Informe Anual de Actividades 1995, Buenos Aires, CNEA, 1996 y CNEA. Informe Anual de Actividades 1997, Buenos Aires, CNEA, 1998.

de privatización para las Centrales Nucleares. Pero además, la legislación apuntaba a dividir y reagrupar las funciones de la CNEA en tres nuevas unidades a fin de facilitar el proceso de venta y la finalización de Atucha II por capitales privados.

Al igual que en otras ramas del sector eléctrico, la decisión fue tomada unilateralmente por el poder Ejecutivo sin tomar en consideración la propuesta realizada por el entonces Presidente de la CNEA, Manuel Mondino. Según se comentaba entonces, Mondino había sugerido la división de la CNEA en dos empresas. Por un lado, se crearía una Central reguladora, que funcionaría autárquicamente y retendría la parte de la investigación, con 500 de los 4800 empleados existentes; un "holding" empresarial conformado por el resto de las actividades, susceptible de ser vendido en acciones en bloque o subdivididas por actividad pero regulado por la Central Reguladora. Pero además, había solicitado que no se modificaran las partidas presupuestarias asignadas a la 
CNEA, así como también afirmaba que la institución debía asegurarse la prerrogativa de finalizar Atucha II. ${ }^{26}$

La propuesta de Manuel Mondino, si bien no fue considerada por el gobierno de turno, apuntaba a mantener el rol rector de la CNEA sobre las empresas así como la interrelación de las mismas en torno al ciclo de combustible y las Centrales Nucleares.

La política neoliberal efectivamente implementaba, apuntó en cambio a dividir verticalmente el sector y desvincular la actividad de generación de la de regulación e investigación. Al igual que en otras ramas del sector eléctrico, el decreto 1540/1994 planteaba unilateralmente la participación de la CNEA en tres nuevas entidades;

- Un ente Regulador (ENREN) que funcionaría de forma autárquica y se encargaría de fiscalizar y regular la actividad.

- Una Sociedad Anónima (NASA) con totalidad de acciones en el Estado Nacional que se operaría las Centrales Atucha I y Embalse y se encargaría de la construcción, puesta en marcha y operación de la Central Atucha II hasta la privatización.

- Una "CNEA Residual" que solo retendría las funciones de formación de recursos humanos, investigación y desarrollo de tecnologías.

26. BORCHES, Carlos.,"CNEA: el negocio y la investigación”, en Microsemanario, Buenos Aires, 1994, V. 4, N¹59. Disponible en: <http://www.fcen.uba.ar/ prensa/micro/1994/ms159> [Consulta: 3 de Marzo 2014].
Ignorando la compleja interrelación de todos los componentes del sector, el decreto 358/97 sancionado algunos años después, exoneraba al futuro comprador de las Centrales Nucleares de comprar insumos a las empresas creadas por la Comisión. Históricamente, hemos señalado que la CNEA había asegurado el abastecimiento de minerales, concentrado de uranio y demás elementos combustibles fabricados en el país a través de la creación de empresas mixtas. Esta situación era blanco de críticas para quienes consideraban que "los costos de las construcciones y aún de la explotación de las actividades productivas están engrosados por las transferencias de fondos orquestadas mediante una compleja trama de contrataciones con la propia CNEA y sus múltiples empresas satélites". ${ }^{27}$ Sin embargo, según la filosofía de la institución que hemos desarrollado en el capítulo anterior, esta situación permitía al Plan Nuclear un mayor grado de autonomía frente a las presiones del Club de Londres, sobre todo en materia de combustible utilizado para las Centrales Nucleares.

De esta forma, se ponía en duda la supervivencia misma de la cadena productiva del ciclo de combustible, en la cual Atucha I y Embalse resultaban eslabones fundamentales. $\mathrm{La}$ eventual decisión por parte del comprador de no adquirir esos insumos en el país, produciría la quiebra de estas empresas y una sustancial pérdida del conocimiento tecnológico adquirido durante los últimos años. En forma paralela, circuló en el Ministerio de Economía la posibilidad de cerrar CONUAR S.A., privatizar DIOXITEK S.A. y obtener los

27. GABINETE PARALELO, "Informe Especial. Organización Institucional”, en Gabinete Paralelo, s/d, 1991, p.19. 
combustibles de proveedores externos, aunque finalmente no hubo avances en este sentido. ${ }^{28}$

\section{La trayectoria seguida por el complejo empresarial}

De la información presentada se desprende que, efectivamente existía una política clara y sostenida que apuntaba a descentralizar las funciones de la Comisión, tanto en un sentido geográfico como también operativo. Esta decisión manifestaba, por un lado, la importante cuota de autonomía con que la institución se manejaba respecto de otras áreas burocráticas del Estado nacional. Por otra parte, resultaba sintomática de una temprana conciencia que sus actores habían desarrollado acerca de cuestiones que iban más allá de la cuestión nuclear; el desarrollo científico autónomo, el fortalecimiento de la empresa nacional y la vinculación entre ambas esferas.

La paralización del Proyecto Atucha II hacia mediados de los '90 significó un duro golpe para el eslabonamiento productivo que se había generado en torno a la obra. En tanto, la demanda de las Centrales ya existentes, Atucha I y Embalse, no alcanzaba para mantener vivo el sector en el largo plazo. Esta situación obligó a las empresas a diversificar su oferta de productos y servicios para sobrevivir, así como también impulsó la búsqueda de nuevos mercados dentro y fuera del país. $\mathrm{El}$ análisis de la trayectoria seguida por las industrias nucleadas en torno a la CNEA durante esos años, enmarcadas en un contexto de apertura económica, privatizaciones y desindustrialización, demuestra que solo

28. HURTADO DE MENDOZA, Diego, El sueño de la Argentina Atómica..., Op. Cit., p. 285. tuvieron oportunidad de éxito aquellas que lograron reorientarse a otras actividades productivas que no estaban directamente relacionadas con el Plan Nuclear y la Construcción de las Centrales.

El primero de los emprendimientos en sufrir los embates de la privatización, fue la Empresa Nuclear Argentina de Centrales Eléctricas S.E. (ENACE S.E.). Constituida mediante un acuerdo entre la CNEA y la empresa alemana Siemens KWU, la entidad se hallaba ligada directamente a la arquitectura, diseño, ingeniería y gestión de las Centrales Nucleares programadas por el Plan Nuclear de 1979. La demora creciente del proyecto Atucha II, la imposibilidad de pagar los insumos comprados a Siemens, y la inflación crónica del país se habían traducido también en un aumento sustancial de los costos: si inicialmente se preveían entre 1500 y 1700 millones de dólares totales, para 1994 la empresa ENACE S.E. llevaba invertidos 2000 millones y se estimaban 400 más para finalizar la obra. Dos años después, se estimaba que la deuda total de ENACE ascendía a $\$ 36.238 .576$ pesos..$^{29} \mathrm{~A}$ esta situación, se sumaba la incertidumbre respecto del futuro de la privatización de las Centrales que nunca pudo concretarse. Finalmente, a mediados de 1996 se procedió a liquidar la entidad y trasladar lo que quedaba de sus activos y personal a la empresa Nucleoeléctrica Argentina S.A., creada en 1994 a partir de la reorganización administrativa de la CNEA.

29. ROSSI, Antonio, "Atucha II se queda sin fondos", en Diario Clarin, Buenos Aires, 01/12/1996. Disponible en: <http://edant.clarin.com/diario/96/12/01/O03001d.htm> [Consulta: 3 de Marzo 2014] y ENACE, Relevamiento del Estado patrimonial de activos y pasivos ciertos y contingentes al 31/12/96, Documento Interno, Buenos Aires, 1996. 
Otro tanto sucedió con la empresa NUCLEAR MENDOZA S.E., creada mediante un contrato que ligaba a la Comisión y la Provincia de Mendoza en torno a la prospección de minerales uraníferos. Si bien en este caso la Comisión no poseía capital accionario en la entidad, retenía la prerrogativa de designar a los miembros del Directorio. Luego de la paralización del Plan Nuclear, la empresa había intentado diversificar sus actividades realizando servicios de irradiación $o$ instalando un observatorio de rayos cósmicos. $^{30}$ Sin embargo, en el marco del retroceso del sector nucleoeléctrico y la baja demanda de combustibles uraníferos que se derivaba de esta situación, su misión original carecía de sentido. Finalmente, el contrato debió rescindirse en julio del 2001 dejando sin efecto la existencia de la entidad.

Dos casos menos estudiados son las empresas ALTEC S.E. y CORATEC S.E. Al igual que la empresa Nuclear Mendoza, se trataba de emprendimientos en los que la CNEA no poseía participación accionaria, sino que se reservaba el poder de designar a algunos de los miembros del Directorio.

En el primer caso, se trataba de una empresa originalmente dedicada al desarrollo de servicios informáticos para las Centrales Nucleares. La entidad fue creada en 1985 por medio de un convenio con la provincia de Río Negro, quien aportaría los recursos económicos, y la CNEA, encargada de proveer recursos humanos y equipos. Este contrato debía renovarse cada cinco años. En junio de 1995, se declaró la intervención de la empresa a pesar de que dicho proceso finalmente nunca

30. CNEA, Memoria Anual 2001, CNEA, Buenos Aires, 2002, p. 9. pudo llevarse a cabo. ${ }^{31}$ Actualmente, la entidad continúa existiendo sin la participación de la Comisión. Sin embargo, ha diversificado notoriamente la naturaleza de los servicios ofrecidos en materia de seguridad y control de acceso, desarrollo de software, telefonía y redes de comunicación. Incluso ha desarrollado sistemas en pequeña escala de urnas electrónicas. ${ }^{32}$ Además, se encarga de brindar servicios a varias reparticiones de gestión estatal (ministerios, provincias y agencias), así como también empresas regionales (INVAP S.A., Trancomahue, entre otras).

Por otra parte, CORTAEC S.E. fue constituida mediante un contrato con el gobierno provincial cordobés para producir radioisótopos en esa región. En este caso, no existe documentación oficial que esclarezca los motivos de la disolución de la sociedad cuya liquidación finalizó en julio de 1999. ${ }^{33}$

E1 resto de las entidades, en cambio, logró superar la coyuntura crítica y actualmente forman parte del panorama institucional argentino. En este punto, cabe destacar el uso de dos estrategias para asegurar la supervivencia durante la paralización del Plan Nuclear; el desarrollo de nuevos productos y el desarrollo de nuevos mercados, sobre todo en el exterior. De esta forma, gran parte de las empresas que analizaremos a continuación alcanzaron nuevos niveles de diversificación combinando ambas estrategias a través de la reutilización de recursos y capacidades

31. DECRETO Provincial N 777/1995, Boletin Oficial de la Provincia de Río Negro, 23 de junio de 1995.

32. Disponible en: <http://www.altec.com.ar/index. php?catID $=843>$ [Consulta: 10 de Agosto 2014]

33. LEY Provincial N ${ }^{\circ} 8771$, Boletín oficial de la Provincia de Córdoba, 12 de julio de 1999. 
existentes (negocios relacionados) o la creación de nuevas cadenas de valor (negocios no relacionados).$^{34}$ Asimismo, este trabajo no ignora una cuestión no desdeñable pero difícil de documentar, que tiene que ver con margen de actuación política con que en su momento se desempeñaron los gobernadores de las distintas provincias involucradas en relación al poder ejecutivo. En general, aquellos mandatarios alineados con el menemismo pudieron obtener mayores concesiones que permitieron la supervivencia de algunos de estos emprendimientos. Es el caso del gobernador Luis Felipe Sapag en la provincia de Neuquén (1995-1999), quien desde 1995 conformaba el Directorio de ENSI. Dado que el cierre de la planta produciría el despido de entre 400 y 500 personas, la situación fue negociada directamente con presidencia para evitar reclamos. Si bien dicho análisis de índole político no será profundizado en este trabajo, constituye una línea de investigación inexplorada abierta a indagaciones futuras. ${ }^{35}$

El caso más reseñado por la historiografía argentina, ha sido INVAP S.A. ${ }^{36}$ Conformada

34. ANSOFF, Igor, "Strategies for Diversification", en Harvard Business Review, Boston, 1957, V.XXXV, N5, pp. 113-124 y THOMPSON, Arthur, Administración estratégica. Teoría y casos, McGraw Hill, Mexico, 2012.

35. Disponible en: <http://www.sapag.com.ar/index.php/ mi-historia> [Consulta: 3 de Noviembre 2015]

36. Véase: BUCH, Tomás, "INVAP en Australia. Realidades, prejuicios y manipulaciones", en REDES, Bernal, 2002, V.X, N¹9, pp. 135-148; THOMAS, Hernan, VERSINO, Mariana y LALOUF, Alberto, "INVAP: una empresa nuclear y espacial argentina", en THOMAS, Hernan, SANTOS, Guillermo y FRESSOLI, Mariano, Innovar en Argentina. Seis trayectorias empresariales basadas en estrategias intensivas en conocimiento, Lenguaje Claro, Buenos Aires, 2013, pp. 105-150; QUIROGA, Juan Martin y AGUIAR, Diego, "El caso INVAP S.E. en el desarrollo de tecnología de radares en Argentina", ponencia presentada en XXIV en 1976 junto con la provincia de Río Negro, la empresa nació orientada a la industria nuclear y espacial. Su existencia representa una continuación del Programa de Investigaciones Aplicadas (PIA) desarrollado por un grupo de trabajo en la CNEA desde 1971. Durante los años '90, INVAP amplió su rango de actividad hacia el tratamiento de residuos industriales peligrosos a través de la creación de la subsidiaria Ailinco S.A. con un 51\% de participación accionaria. Actualmente, también incorporó tareas orientadas al desarrollo de energías alternativas, defensa, sistemas médicos y telecomunicación digital. El éxito de INVAP tiene que ver con la creación de paquetes tecnológicos de alto valor agregado a través de los cuales ha logrado una inserción exitosa en el mercado externo. A este fin, el $85 \%$ de los recursos humanos que componen la empresa poseen un alto nivel de especialización y la organización del trabajo se realiza a través de la conformación de grupos a los cuales se asignan distintos proyectos. Por otra parte, no solo ha logrado capear la crisis a través del desarrollo de nuevos productos y servicios no relacionados, sino que además ha realizado una significativa cantidad de trabajos para el sector externo. En este punto, destaca la venta de reactores de investigación a Australia (OPAL), Egipto (ETRR-2), Argelia (NUR) y

Jornadas de Historia Económica, Universidad de Rosario, Rosario, 2014; LUGONES, Manuel, “La conformación del sector nuclear en argentina: la creación de la empresa INVAP S.E. y su papel en el desarrollo del sector", en LORENZANO, Pablo (editor) Historias de la ciencia Argentina III, Editorial de la Universidad Nacional de Tres de Febrero, Buenos Aires, 2008, pp. 159-166; KOZULJ, Roberto y LUGONES, Manuel, "INVAP y el desarrollo de una trama de base tecnológica: evolución histórica y situación actual", en DELFINI, Marcelo, DUBBINI, Daniela, LUGONES, Manuel y Rivero, Ivana, (editores) Innovación y empleo en tramas productivas de Argentina, Prometeo, Buenos Aires, 2007, pp. 323-347. 
Perú (RP-0 y RP-10). ${ }^{37}$ Cabe destacar además, que la institución no recibe aportes del Estado, sino que depende exclusivamente de la venta de servicios. Por este motivo, sus prácticas empresariales son similares a las de las firmas privadas $\sin$ fines de lucro. ${ }^{38}$

En esta misma línea se ubica FAE S.A., constituida en 1986 como empresa subsidiaria de CONUAR S.A. con participación de la CNEA. Originalmente fue fundada para fabricar vainas y barras de circonio para su utilización en el reactor de las Centrales Nucleares. En los años que siguieron al desmantelamiento del Plan Nuclear, la empresa diversificó su producción hacia la fabricación de aleaciones especiales, sobre todo tubos de titanio y níquel para la industria aeroespacial y aeronáutica. Dado que actualmente es el único productor de tubos sin costuras a nivel latinoamericano, en los últimos años ha concretado ventas en Brasil, Uruguay, Chile, Bolivia, México, Venezuela y Colombia. También ha exportado productos a Estados Unidos, Corea, Sudáfrica, España, Reino Unido, Bélgica. ${ }^{39}$

En menor medida, la empresa ENSI S.E. puede equipararse a las anteriores. Fundada en 1989 mediante la asociación de la CNEA y la provincia de Neuquén, su misión consistía en producir y comercializar agua pesada para los reactores argentinos. Hacia 1995, desarrolló

37. Disponible en: <http://www.invap.com.ar/es/areanuclear-de-invap/proyectos.html> [Consulta: 4 de Agosto 2014].

38. THOMAS, Hernan, VERSINO, Mariana y

LALOUF, Alberto, “INVAP: una empresa nuclear...”, Op. Cit., p. 107.

39. Disponible en: <http://www.fae.com.ar/fr_default. asp?txtLink=NuestraEmpresa.html> [Consulta: 4 de Agosto 2014].

84 nuevos productos y servicios no relacionados en la rama de ingeniería, inspección y mediciones ambientales para otras fuentes de producción energética, sobre todo, la industria petroquímica y gasífera del país. Por otra parte, la exportación de agua pesada se concretó con excelentes resultados hacia 1997 mediante tratados comerciales con Corea del Sur, Estados Unidos y Canadá. Actualmente cuenta con el asesoramiento tecnológico y científico de la Comisión, la cual posee un 49\% del capital accionario. ${ }^{40}$

Las tres empresas reseñadas representan los casos en que primaron ambas estrategias; desarrollo de nuevos productos y servicios e integración de nuevos mercados. En cambio, DIOXITEC S.A. y CONUAR S.A. han priorizado solo una de estas variables.

En el caso de CONUAR S.A., se trata de una empresa fundada en 1981 por la CNEA en consorcio con la firma Pérez Companc S.A. Con un 33\% del capital accionario en manos de la Comisión, la finalidad de la empresa consistía en fabricar elementos combustibles para las Centrales Nucleares y los reactores de investigación, pastillas de uranio natural y levemente enriquecido y núcleos de cobalto. A diferencia de su subsidiaria, FAE S.A., CONUAR no diversificó su producción en los años que siguieron a la paralización del Plan Nuclear. En cambio, optó por la estrategia de integración en el mercado externo a través de la venta de combustibles en Europa, Canadá y Brasil. ${ }^{41}$

\footnotetext{
40. Disponible en: <http://www.ensi.com.ar/docs/ aguapesada/fr-aguapesada.html> [Consulta: 4 de Agosto 2014].
}

41. Disponible en: <http://www.conuar.com/home.htm> [Consulta: 10 de Agosto 2014]. 
Por último, DIOXITEK S.A. fue creada en 1996 para operar la planta de dióxido de uranio para las Centrales Nucleares fundada en 1982. Hasta la constitución de la empresa, dicha actividad había permanecido bajo la órbita de la CNEA. A partir del año 2002, DIOXITEK sumó las tareas de diseño, producción y comercialización de Cobalto ' 60 , insumo básico de la medicina nuclear. ${ }^{42} \mathrm{Sin}$ embargo, la empresa no logró insertarse en el mercado externo. Actualmente, la totalidad de la producción de la empresa se encuentra circunscripta a mercados locales en el ámbito nacional.

En definitiva, cinco de las nueve empresas reseñadas pudo sobrevivir al desmantelamiento del Plan Nuclear durante la década de 1990. Su inserción en el panorama productivo argentino no resulta un dato menor si se tiene en cuenta la influencia negativa del contexto económico y político del país, signado por la paralización de la obra Atucha II y la destrucción generalizada de las industrias nacionales.

\section{Conclusiones}

A lo largo de sus años de existencia, la CNEA se transformó en un referente institucional en materia de desarrollo de tecnología ligada al sector nucleoeléctrico. Sin embargo, en diferentes contextos sus integrantes y directivos percibieron la necesidad de desligar las actividades productivas de la órbita de tareas de I+D. El derrame de dichos conocimientos hacia emprendimientos de origen mixto y estatal constituyó un temprano objetivo de

42. Disponible en: <http://www.dioxitek.com.ar/ cs/270/16368/institucional> [Consulta: 10 de Agosto 2014]. la institución, que comenzó a tomar forma desde mediados de los años '70. Siguiendo esta lógica, el Plan Nuclear se vería beneficiado por el aporte de bienes y servicios que debían ser producidos de forma más ágil y flexible bajo una lógica empresarial, separada del ámbito de la investigación.

Luego del retorno de la democracia, las dificultades financieras obligaron a la institución a profundizar dicha política en pos de asegurar la supervivencia misma del sector. Dada la reducción constante de las partidas presupuestarias asignadas a la CNEA luego de 1984, muchos de sus Directivos consideraban que el despegue de ciertas actividades podría constituir una alternativa para mantener su funcionamiento. La supervivencia de gran parte de esos emprendimientos en años venideros, demostrarían que se trataba de una política atinada.

Por otra parte, es claro que el desmantelamiento posterior del Plan Nuclear y la paralización de sus obras constituyó un severo quiebre para el polo industrial reseñado. Aquellas empresas que dejaron de existir, lo hicieron porque no pudieron insertarse en el mercado fuera de la política nuclear. ENACE, ALTEC, CORATEC y Nuclear Mendoza son ejemplos del fracaso derivado de un contexto político y económico sumamente adverso.

En cuanto a las razones que explican el éxito de algunas de estas empresas, la clave radica en la capacidad de brindar productos y servicios fuera del ámbito nuclear o insertarse en mercados externos. Tanto INVAP, como FAE y ENSI representan ejemplos situados en el extremo más alto de la escala, dado que en ellos se combinaron ambos criterios para asegurar la continuidad de los emprendimientos. En los 
tres casos, las empresas mencionadas logran pasar de tener un único cliente en la CNEA para insertarse luego en mercados dentro y fuera de los límites nacionales. En un nivel intermedio, pueden ubicarse DIOXITEC, CONUAR y ALTEC, las cuales apuntaron a priorizar una de las dos estrategias. En contraposición a los discursos neoliberales, la existencia de un contexto adverso no repercutió en muchas de las firmas con capital netamente estatal. Tanto INVAP como DIOXITEC, representan casos exitosos a pesar de que gran parte de su capital accionario no estaba atado al régimen privado. Por otra parte, en todos los casos se trata de empresas con un alto nivel de valor agregado procedente de desarrollos científicos y tecnológicos autónomos. Además, cuentan con un porcentaje mayoritario de recursos humanos sumamente especializados.

En conclusión, la existencia del polo industrial nucleado originalmente en torno a la CNEA desmiente la idea de que los países periféricos no poseen aptitudes para realizar desarrollos científicos y tecnológicos autónomos y trasladar los resultados hacia el ámbito empresario. A pesar de nacer y consolidarse en un contexto económico y político sumamente inestable, las firmas "exitosas" que sobrevivieron a las políticas neoliberales, lograron capear los avatares del entorno generando resultados sumamente satisfactorios. Un estudio más contundente sobre esta cuestión sería fructífero para rescatar los aprendizajes de dichas trayectorias e indagar en los motivos que posibilitaron su desarrollo exitoso, a fin de aportar valiosas lecciones al diseño de políticas públicas en nuestro país.

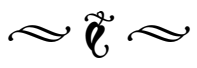




\section{Bibliografía}

ALBORNOZ, Mario y GORDON, Ariel, "La política de ciencia y tecnología en Argentina desde la recuperación de la democracia (1983-2009)”, en ALBORNOZ, Mario y JESÚS Sebastián (editores) Trayectorias de las politicas cientificas y universitarias de Argentina y España, CSIC, Madrid, 2011.

ANSOFF, Igor, "Strategies for Diversification", en Harvard Business Review, Boston, 1957, V. XXXV, N 5, pp. 113-124.

BELINI, Claudio y ROUGIER, Marcelo, El Estado Empresario en la Industria Argentina. Conformación y crisis, Manantial, Buenos Aires, 2008.

BORCHES, Carlos, “CNEA: el negocio y la investigación”, en Microsemanario, Buenos Aires, 1994, V. IV, $\mathrm{N}^{\circ}$ 159. Disponible en: <http://www.fcen.uba.ar/prensa/micro/1994/ms159> [Consulta: 3 de Marzo 2014].

BOTANA, Natalio, SABATO, Jorge, "La ciencia y la tecnología en el desarrollo futuro de América Latina”, en SABATO, Jorge (compilador) El Pensamiento Latinoamericano el la problemática Ciencia, Tecnología, Desarrollo, Dependencia, Paidós, Buenos Aires, 1975, pp. 143-154.

BUCH, Tomás, "INVAP en Australia. Realidades, prejuicios y manipulaciones”, en REDES, Bernal, 2002 V.X, N¹9, pp. $135-148$.

CANITROT, Adolfo, "La disciplina como objetivo de la política económica. Un ensayo sobre el programa económico del gobierno argentino desde 1976”, en Desarrollo Económico, Buenos Aires,1980, V. XIX, , $\mathrm{N}^{\circ} 76$, pp. 453-476.

CASTRO MADERO, Carlos y TAKACS, Esteban, Politica nuclear argentina ¿̨avance o retroceso?, Instituto de publicaciones navales, Buenos Aires, 1991.

ENRIQUEZ, Santiago, “A 50 años del Servicio de Asistencia Técnica a la Industria (SATI): Apuntes de una heterodoxia", en Revista de la CNEA, Buenos Aires, 2011, V.XI, N41-42, pp. 14-20. Disponible en: <http://www.iaea.org/inis/collection/NCLCollectionStore/_Public/46/135/46135001.pdf>.

FREDERIC, Sabina, Las trampas del pasado: las Fuerzas Armadas y su integración al Estado democrático en Argentina, Fondo de Cultura Económica, Buenos Aires, 2013.

GABINETE PARALELO, “Informe Especial. Organización Institucional”, en Gabinete Paralelo, s/d, 1991.

HURTADO DE MENDOZA, Diego, La ciencia argentina. Un proyecto inconcluso: 1930-2000, Edhasa, 
Buenos Aires, 2010.

HURTADO DE MENDOZA, Diego, El sueño de la Argentina Atómica. Politica, tecnología nuclear y desarrollo nacional (1945-2006), Edhasa, Buenos Aires, 2014.

KOSACOFF, Bernardo (editor) Estrategias empresariales en tiempos de cambio. El desempeño industrial frente a nuevas incertidumbres, Universidad Nacional de Quilmes/CEPAL, Bernal, 1998.

KOZULJ, Roberto y LUGONES, Manuel, "INVAP y el desarrollo de una trama de base tecnológica: evolución histórica y situación actual”, en DELFINI, Marcelo, DUBBINI, Daniela, LUGONES, Manuel y Rivero, Ivana (editores) Innovación y empleo en tramas productivas de Argentina, Prometeo, Buenos Aires, 2007, pp. 323-347.

LUGONES, Manuel, "La conformación del sector nuclear en argentina: la creación de la empresa INVAP S.E. y su papel en el desarrollo del sector", en LORENZANO, Pablo (editor) Historias de la ciencia Argentina III, Editorial de la Universidad Nacional de Tres de Febrero, Buenos Aires, 2008, pp. 159-166.

OTEIZA, Enrique, “Introducción”, en OTEIZA, Enrique (et al.) La politica de investigación científica y tecnológica argentina. Historia y perspectivas, CEAL, Buenos Aires, 1992, pp. 11-17.

OTEIZA, Enrique, "Drenaje de Cerebros. Marco histórico y conceptual”, en REDES, Bernal, 1996, V. III, N7,pp.101-120.Disponible en:<http://iec.unq.edu.ar/images/redes/RedesN07/Artculos/Perspectivas/ Drenaje\%20de\%20cerebro\%20Marco\%20histrico\%20y\%20conceptual\%20Enrique\%20O.pdf>.

QUIROGA, Juan Martin y AGUIAR, Diego, "El caso INVAP S.E. en el desarrollo de tecnología de radares en Argentina", ponencia presentada en XXIV Jornadas de Historia Económica, Universidad de Rosario, Rosario, 2014.

RAMÍREZ, Hernán, Corporaciones en el poder. Institutos económicos y acción politica en Brasil y Argentina: IPÊS, FIEL y Fundación Mediterránea, Lenguaje Claro, Buenos Aires, 2007.

RODRIGUEZ, Milagros, "Cuentas Pendientes. La producción historiográfica argentina en torno a la generación nucleoeléctrica”, ponencia presentada en XLI Reunión Anual de la AATN, Ministerio de Relaciones Exteriores y Culto, Ciudad Autónoma de Buenos Aires, diciembre de 2014.

SÁBATO, Jorge, "Quince años de metalúrgia en la Comisión Nacional de Energía Atómica [Entrevista]", en Ciencia Nueva, 1973, N 15, pp. 1-19.

SARALE, Gustavo, Poder económico y economistas: El papel de la Fundación Mediterránea, la FIEL y el CEMA en la construcción de la hegemonia neoliberal en Argentina, 2006. Disponible en: <http://www.fcp.uncu. 
edu.ar/upload/PODERECONOMICOYECONOMISTAS.PDF> [Consulta: 15 de Mayo 2014].

SCHORR, Martin, Industria y Nación. Poder económico, neoliberalismo y alternativas de reindustrialización en la Argentina contemporánea, Edhasa, Buenos Aires, 2004.

THOMAS, Hernán, SANTOS, Guillermo y FRESSOLI, Mariano, Innovar en Argentina. Seis trayectorias empresariales basadas en estrategias intensivas en conocimiento, Lenguaje Claro, Carapachay, 2013.

THOMAS, Hernan, VERSINO, Mariana y LALOUF, Alberto, "INVAP: una empresa nuclear y espacial argentina”, en THOMAS, Hernan, SANTOS, Guillermo y FRESSOLI, Mariano, Innovar en Argentina. Seis trayectorias empresariales basadas en estrategias intensivas en conocimiento, Lenguaje Claro, Buenos Aires, 2013, pp. 105-150.

THOMPSON, Arthur, Administración estratégica. Teoría y casos, McGraw Hill, Mexico, 2012. 